Service social

\title{
Service social des groupes en contexte d'autorité
}

\section{Beulah Rothman et Catherine Papell}

Volume 39, numéro 1, 1990

Le groupe ici et ailleurs

URI : https://id.erudit.org/iderudit/706456ar

DOI : https://doi.org/10.7202/706456ar

Aller au sommaire du numéro

Éditeur(s)

École de service social de l'Université Laval

ISSN

1708-1734 (numérique)

Découvrir la revue

Citer cet article

Rothman, B. \& Papell, C. (1990). Service social des groupes en contexte d'autorité. Service social, 39(1), 45-63. https://doi.org/10.7202/706456ar

\section{Résumé de l'article}

Face à l'augmentation troublante de l'hostilité dans notre monde contemporain, qui se traduit par la désorganisation sociale, l'escalade de la violence et l'augmentation des injustices, on peut se demander si le service social des groupes peut être adapté à notre milieu actuel sans que l'essence de ses valeurs et de ses habiletés soit sacrifiée. Les auteures analysent le travail de groupe en contexte d'autorité. C'est précisément dans de tels cadres que les praticiens du groupe sont confrontés aux réalités sociales les plus amères et les plus nocives de notre société et que leur méthode est directement mise en cause. 
Beulah Rothman, professeure à l'École de service social, Barry University, Miami Shores, Floride.

Catherine Papell, professeure à l'École de service social, Adelphi University, Garden City, New York.

\section{Service social des groupes en contexte d'autorité ${ }^{1}$}

Beulah Rothman Catherine Papell

La fin de la décennie 80 voit s'instaurer un climat qui, pour ceux qui le perçoivent, "fait craindre pour l'humanité ». Ces mots sont utilisés par I'historienne Barbara Tuchman (1982 : 13) pour rappeler les événements de 1914. Pour nous, ils évoquent l'augmentation troublante de l'hostilité dans notre monde contemporain, traduite par la désorganisation sociale, l'escalade de la violence et l'augmentation des injustices. Nous nous demandons si le service social de groupe - méthode issue d'une idéologie humanitaire dont la pratique s'inspire de celle des institutions à caractère démocratique - peut être adapté à notre milieu actuel sans que l'essence de ses valeurs et de ses habiletés soit sacrifiée.

Nous répondons, dans le présent exposé, à la demande que nous avons reçue d'analyser le travail de groupe en contexte d'autorité. C'est précisément dans de tels cadres que nous sommes confrontés aux réalités sociales les plus amères et les plus nocives de notre société actuelle et que notre méthode est directement mise en cause.

Des historiens comme Tuchman (1982 : 25) peuvent s'interroger sur la pertinence d'écrire l'histoire pendant qu'elle est encore chaude. Contrairement aux historiens, nous les professionnels des services humains, n'avons pas le loisir de prendre du recul. Nous devons, dans l'exercice de nos rôles de praticiens directs, faire constamment face aux dilemmes immédiats et aux réalités qui, sans cesse, mettent à jour notre fragilité autant que notre force. 
Dans le contexte de services exigés par un tribunal ou en application d'une politique de bien-être social, la tradition reconnue du travail social de groupe est mise à l'épreuve. Bernstein (1988) définit cette tradition en ces termes ${ }^{2}$ : «Un respect des humains qui exige des occasions favorables pour que chacun prenne ses propres décisions, qu'elles soient sages ou insensées. [...] Une société qui respecte ses citoyens et qui leur permet de prendre librement leurs décisions est grandement souhaitable et se situe à un échelon élevé de l'éthique. Cette possibilité de décider librement constitue un moyen d'évaluation des sociétés. "

L'acquisition de l'autonomie du groupe et de ses membres dans la prise de décision a aussi servi de critère pour évaluer le service social de groupe et le succès de sa méthode (Lang, 1986; Papell et Rothman, 1980). Lang (1972) définit le groupe autonome ayant atteint son plus haut degré de développement comme étant celui qui parvient à l'autorégularisation des initiatives interpersonnelles et au partage de son leadership. Dans une étude ultérieure (1986), elle utilise une fois de plus ces paramètres pour caractériser la collectivité, forme sociale qui ne parvient jamais à l'autonomie. L'autonomie de groupe et la liberté de prise de décision peuvent être actuellement en péril ou peuvent, pour le travailleur social en contexte d'autorité, sembler peu réalistes ou inappropriées aux tâches à accomplir.

La fonction du travailleur social de groupe qui consiste à maîtriser le conflit, à resocialiser les clients vers un comportement plus responsable et plus bienveillant, à faciliter le changement chez les membres violents ou abuseurs est connue et bien établie (Garvin, 1988). Ce qui caractérise le contexte d'aujourd'hui est l'agrandissement du cadre des services de groupe et l'omniprésence d'un contexte d'autorité dans lequel s'insèrent ces services. De plus en plus, le praticien peut trop facilement se voir attribuer un rôle antipathique, tant par les membres que par l'agence elle-même. La tâche du travailleur social de groupe qui exerce un rôle d'autorité imposé de l'extérieur est d'affronter les ambiguïtés et les dilemmes sans renoncer à son engagement professionnel lié aux valeurs démocratiques et à ce qu'elles sous-tendent de liberté de choix, d'activité autonome, d'absence de cœrcition et de changement automotivé.

\section{Vision traditionnelle de l'autorité en travail de groupe}

Dans l'histoire du service social de groupe, le concept d'autorité a été lié au rôle du professionnel en sa qualité d'expert et à sa fonction 
de leadership. Les questions d'autorité ont été traitées comme des phénomènes inhérents aux processus internes du groupe principalement centrés sur l'interaction entre le professionnel et les membres du groupe. L'influence du professionnel est en grande partie tributaire de sa compétence, de ses connaissances et de son rôle dynamique dans la formation du groupe. Cette forme d'autorité doit cependant être gagnée par le professionnel et lui être conférée par le groupe (Schwartz, 1961). Ephross et Vassil (1988: 113) soulignent aussi que " au début, le capital autorité des leaders se situe généralement du côté positif de la balance ».

La conception de l'autorité, même associée au rôle du travailleur social à titre de porte-parole des valeurs et des normes sociales (Vinter, 1974), soulève de façon constante et parallèle, une préoccupation au sujet de l'autodétermination. Les normes morales et d'éthique professionnelle ont protégé le praticien d'une utilisation abusive de son autorité.

Une autre raison, pour le service social professionnel de groupe, de s'intéresser à l'autorité, provient des enjeux liés à ce qu'il est convenu d'appeler la "crise d'autorité " (Levine, 1979) et à ceux associés à "la phase de pouvoir et de contrôle " (Garland, Jones et Kolodny, 1965) soulignés dans les modèles de développement de groupes. Ces modèles d'épigénèse laissent croire qu'en général, il se produit une certaine redistribution du pouvoir entre le professionnel et les participants, ce qui constitue une dynamique importante vers un nouveau degré de maturité du groupe. Le travailleur social s'attend normalement à céder de plus en plus d'autorité au groupe.

Les études théoriques sur le travail social de groupe n'ont pas approfondi le concept d'autorité tiré du mandat d'une agence. Le travail d'Hasenfeld (1985) fait exception et révèle des idées importantes sur le contexte organisationnel du travail de groupe et sur I'influence qu'il exerce sur la pratique. Charles Garvin (1988) a poussé plus avant l'étude du contexte organisationnel; il a fourni une contribution importante à la compréhension de la pratique du travail social de groupe dans un cadre de contrôle social. Le modèle qu'il a mis au point, en utilisant un concept charnière de fonction organisationnelle, permet d'envisager l'autorité comme étant issue de facteurs liés à l'agence plutôt que de la seule dynamique du groupe ou de celle des individus.

Au cours des dernières années, un certain nombre d'études cliniques et de livres portant sur des populations et des problèmes sociaux particuliers ont fourni un éclairage supplémentaire à la pratique du travail social de groupe dans un contexte d'autorité (par exemple Beninati, 1989; Brekke, 1989; Brennen et Weakland, 1983; Breton, 1979; Citron, 1978; Farley et Magill, 1988; Finn, 1985; Geller, 
1978; Glassman et Kates, 1982; Lange, Barton et Lanham, 1981; Levine et Gallogly, 1985; Milton, 1981; Nosko et Wallace, 1988; Prudy et Nickle, 1981; Shields, 1986; Smith, 1985).

\section{Dimensions importantes du contexte d'autorité}

On peut trouver un contexte d'autorité dans des organismes et des agences comme les établissements correctionnels, les cliniques de désintoxication, les programmes de traitement d'inceste et de violence familiale et les agences de protection de l'enfance. Quatre caractéristiques de base, inhérentes à ce contexte, peuvent être signalées dans la pratique de groupe :

1. I'assistance obligatoire aux réunions de groupe;

2. une exigence explicite de changement ou de maîtrise du comportement;

3. la soumission des participants et du professionnel à un système de règles officielles, de règlements et de rituels;

4. l'existence d'un système complexe d'intervenants chargés de la surveillance et de l'application des règles et des règlements et de veiller à ce que les buts soient atteints.

Même si l'importance de ces quatre caractéristiques varie selon les milieux, on peut constater que toutes sont présentes à différents degrés lorsque le rôle d'autorité déléguée est sanctionné et utilisé par l'organisme.

Ces quatre dimensions seront étudiées dans le but d'approfondir notre compréhension de leurs propriétés et de leurs effets sur le groupe, sur ses membres et sur le praticien. L'étude des interventions et des stratégies mettra l'accent sur la promotion du processus de prise de décision, sur l'établissement des buts, de même que sur la réflexion critique et l'action du groupe et de ses membres. Des stratégies orientées vers le changement organisationnel, comme le plaidoyer au nom des membres, la négociation avec les personnes responsables des décisions et la: persuasion des autorités influentes seront aussi exposées.

\section{Assistance obligatoire aux réunions de groupe}

L'obligation d'assister aux réunions de groupe peut provenir de différentes sources allant de l'ordonnance jusqu'à des formes plus subtiles de contrainte. Un juge peut suspendre ou réduire l'incarcération d'un détenu s'il participe à un counseling individuel ou de groupe (Berliner, 1987). On peut demander à un délinquant qui purge une sentence dans un établissement correctionnel de participer à un 
programme de réadaptation. La participation à certains groupes volontaires peut ne pas être exigée par la loi mais la décision d'une personne de ne pas se joindre à ces groupes peut avoir de sérieuses conséquences sur sa mise en liberté, sur le recouvrement de son permis de conduire, sur la fin de sa période de probation ou sur son maintien dans un programme de traitement. C'est ainsi que dans un hôpital psychiatrique (Nightengale, 1989), un groupe de patients est responsable de l'évaluation et des recommandations relatives aux congés accordés. Le patient doit donc participer au groupe pour que sa demande de congé soit étudiée. Ce programme, bien qu'innovateur, utilise le refus de participer comme une indication que le patient n'est pas prêt à être libéré ou que la planification de sa sortie doit être retardée. L'obligation de participer peut, de prime abord, se manifester sous différentes formes mais au fond, la cœrcition est présente dans un contexte d'autorité.

La durée de la participation exigée peut varier mais elle est souvent établie par une ordonnance rigide qui fait suite au délit et n'est pas affectée par des circonstances atténuantes ou par les nécessités de traitement. La personne n'a aucun choix et le professionnel n'a que peu ou pas de pouvoir discrétionnaire pour modifier la participation obligatoire aux activités du groupe. La participation obligatoire ne garantit pas l'établissement d'un groupe fermé et d'un nombre stable de participants. Dans les programmes communautaires en particulier, les groupes sont constitués " d'un flot intermittent de nouveaux membres »(Berliner, 1987 : 13) faisant appel aux habiletés d'intervention utilisées dans les groupes ouverts.

Une durée indéterminée et non négociable d'assistance obligatoire à l'intérieur d'un cadre très structuré peut, en certains cas, fournir l'occasion d'offrir un traitement thérapeutique utile pour certains membres. Une mobilisation d'énergie qui rend les membres plus ouverts au changement, peut être engendrée par la crise (Panepinto, Garrett, Willford et Priebe, 1982). Garvin (1989 : 255) établit que « des mesures énergiques pour pousser les membres à des activités que le professionnel croit susceptibles d'améliorer et même de sauver la vie de la personne » peuvent être justifiées.

Le pouvoir et l'impuissance demeurent, pour les membres comme pour le professionnel (Schulman, 1988), les enjeux cruciaux de la dynamique du groupe qui ont cours dans un contexte d'autorité. Comme on peut s'y attendre, les sentiments troublés et déformés que les membres ont, au cours d'étapes antérieures de développement, éprouvés envers le pouvoir et l'autorité, seront exacerbés par l'imposition de la présence obligatoire. De plus, la présence obligatoire étiquette les membres comme déviants, ce qui renforce les perceptions déficientes ou détériorées du soi. De façon caractéris- 
tique, les membres ne se rendent pas compte des choix ou des solutions de rechange qui ont pu se présenter dans leur vie; ils répondent avec colère et blâment les autres et la société de ce qui leur arrive. Pour eux, il ne fait aucun doute qu'un caprice du destin les a placés dans une telle situation puisqu'ils perçoivent le comportement $d^{\prime}$ autres personnes comme étant plus grave que le leur.

La rage latente ou manifeste des membres sera un défi formidable pour l'intervenant dont la responsabilité est de faciliter le processus par lequel ces personnes récalcitrantes peuvent évoluer vers un degré $d^{\prime}$ interaction responsable et de croissance personnelle que le groupe peut leur offrir. Les membres s'efforceront de réduire l'avantage du pouvoir détenu par le professionnel et l'agence (Emerson, 1962). Les réponses caractéristiques pourront être: 1) la simulation d'une identification à l'autorité du professionnel; 2) la projection d'autres sources de gratification qui existent à l'extérieur du groupe comme étant le rejet de ce que le travailleur social et le groupe peuvent offrir; 3) la formation d'un front d'opposition pour déranger le groupe et réduire le pouvoir du professionnel; 4) l'élaboration d'un bloc d'opposition indifférenciée, orientée vers toute autorité perçue par les membres; 5) l'adoption d'un rôle impersonnel qui se traduit par une impassibilité et une totale apathie.

Reconnaissant l'intensité des sentiments d'oppression et d'impuissance qu'éprouvent les membres, le travailleur social devra répondre immédiatement à cette tension émotionnelle s'il veut établir une communication avec eux. Le professionnel devra, sans détour, ouvertement et sans s'excuser, traiter de l'obligation qu'ont les membres de participer au groupe. Ces derniers doivent se soumettre à cette obligation et répondre de leur comportement à cet égard. La légitimité des réactions des membres est aussi une réalité à laquelle on doit accorder un temps suffisant "d'aération » dans le groupe, et le professionnel doit démontrer qu'il peut écouter et qu'il écoutera l'expression des sentiments des membres à ce propos. À sa manière, le praticien peut faire comprendre au groupe qu'ensemble ils peuvent trouver une solution qui leur permette un choix. "Si chacun fait sa part, on peut tirer quelque chose de positif au fait de se trouver ici ».

L'aisance de l'intervenant dans son rôle d'autorité est primordiale au cours de ces premières discussions. Il doit, pour lui-même et pour le groupe, établir la distinction entre la loyauté à accorder à l'autorité de l'organisme et son soutien à des prises de décision que son groupe pourrait avoir l'occasion de prendre et qui excèdent les limites de son mandat. L'ambiguïté ou la clarté de la position du praticien fait entrevoir aux membres que toute autorité n'est pas absolue. Autrement dit, le mur infranchissable de l'absence de choix qui se dressait devant eux s'écroule. Toutefois, l'obligation de participer demeure et le professionnel l'accepte. 
La réponse du praticien à la rigidité inhérente à l'organisme peut comporter la recommandation de solutions autres que la participation au groupe lorsque celle-ci est nettement contraire à l'intérêt de la personne ou du groupe. Les négociations d'un changement d'horaire par exemple peuvent s'articuler de façon plus efficace avec les changements visés et, par ricochet, démontrer aux membres qu'il est possible de modifier l'absolutisme apparent de l'autorité.

II est important, pour le professionnel qui doit travailler avec un groupe formé de membres soumis à l'obligation de participer, de préciser avec l'organisme les conditions préalables à sa mise sur pied : la taille du groupe, l'espace suffisant pour les rencontres, la disponibilité et l'importance des ressources et un certain contrôle sur le choix des participants.

Finalement, on pourrait - à titre d'intervention stratégique visant à diminuer la pression que cause l'assistance obligatoire recommander la mise en œuvre d'un programme de groupes volontaires. La possibilité de choisir d'autres types d'expériences de groupe pourrait introduire un élément de variété et offrir l'occasion d'une certaine souplesse alors même que les membres luttent contre l'obligation qui leur est imposée.

\section{Exigence explicite de changement ou de maîtrise du comportement}

Les membres de groupes desservis par des organismes où l'autorité est forte ont tendance à n'être pas motivés à modifier les comportements que la société qualifie d'inacceptables et de déviants. Ces personnes sont perçues comme menaçantes pour elles-mêmes et pour les autres, et la société utilise souvent des contraintes légales et correctives pour imposer les changements requis par sa fonction de contrôle social.

Parallèlement à cette fonction dominante de contrôle social, on trouve dans la plupart des contextes d'autorité une idéologie de réadaptation dont le pouvoir est moins grand et les buts moins précis. La coexistence de ces deux fonctions - avec les apparentes incongruités qu'elles véhiculent - imprègne la société plus étendue et ses organismes. Le langage du service social pourrait définir la première comme étant autoritaire et de contrôle et, la seconde, comme humanitaire et de réadaptation.

Le conflit envahissant entre ces deux idéologies opposées est perceptible dans le débat actuel sur la politique sociale relative à la toxicomanie chez les femmes enceintes et le sort déplorable réservé au groupe d'environ 375000 enfants qui consomment du crack. Les solutions possibles, comme le mentionne un article récent du San 
Francisco Chronicle (9 novembre 1989), s'inscrivent dans la fonction de contrôle et comportent différents degrés de réadaptation, d'une part en traitant la mère comme une criminelle qui doit être incarcérée et, d'autre part, en considérant qu'elle peut être réhabilitée si on lui accorde des soins en milieu surveillé. Même si les programmes présentent un aspect de réadaptation plus marqué, ils s'inscrivent dans un contexte d'autorité parce que leur application est obligatoire et que le changement de comportement est exigé.

En contexte d'autorité, les liens entre les aspects de réadaptation humanitaire et de contrôle autoritaire des objectifs du traitement sont souvent obscurs et incongrus; ils reflètent des différences philosophiques profondes dans l'orientation des valeurs, la perception de la réalité et la vision du monde. Lorsque l'aspect de contrôle autoritaire domine de façon excessive, comme dans les établissements correctionnels ou dans des programmes communautaires de désintoxication pour les jeunes, "il règne une atmosphère d'aliénation, de méfiance et d'emprisonnement, lesquels sont de formidables barrières à un traitement efficace. [...] Les clients qui se trouvent dans un tel environnement [...] se renvoient mutuellement l'image d'un comportement déviant [...] qui renforce le comportement antisocial plutôt que celui qui est souhaité "(Feldman, 1985: 475).

L'exemple suivant, tiré d'un dossier d'une agence communautaire pour fugueurs et jeunes drogués, illustre cette contagion négative par rapport aux buts sociaux visés.

La travailleuse sociale dit : "II me semble que la plupart des problèmes sont reliés à la drogue ». Cette phrase a fait rire la plupart des jeunes et ils ont commencé à parler plus haut, tous étant d'accord sur le plaisir que procure la drogue. Gary a parlé le premier : "Ça ne me fait rien. J'aime prendre des drogues. C'est ce qu'il y a de meilleur. J'aime être " high ". Je me sens bien ". J'ai dit : "Gary, je crois comprendre que tu trouves que la drogue est bonne pour toi. Est-ce que tu crois qu'elle te laissera vivre longtemps et en bonne santé ? "Gary m'a répondu qu'il n'en savait rien et que ça ne lui faisait rien. "La drogue lui permet de se sentir bien maintenant " ai-je répondu. «Est-ce qu'il y a autre chose qui te permet de te sentir bien Gary? " II a répondu : "Qu'est-ce qui peut vous faire sentir bien à part la drogue quand vous avez été battu pendant toute votre vie ? Ma mère a été soûle toute sa vie. Elle me battait, me punissait et me donnait des pages et des pages de problèmes de maths à faire encore et encore. Je devais rester assis pendant des heures et des heures à faire ça. Si je me levais elle me battait. Si je lui disais que je ne voulais plus en faire elle le disait à mon père quand il revenait à la maison. Vous voulez voir un poing s'approcher de votre nez - plein de sang partout - les choses sont allées si mal que je me suis enfui. J'aimais mieux être dans la rue que dans cet... endroit ". J'ai fait une pause sans me soucier de ce qu'il me disait. Je dis : "Les choses ont 
dû être très dures pour toi...". Pause. "Je ne puis même pas m'imaginer à quel point les choses ont dû être terribles pour toi... être traité comme ça...". Les jeunes écoutaient attentivement. Certains hochaient la tête comme pour dire : " Je sais, j'ai aussi connu ça ". Je dis : "Gary, avec toutes ces mauvaises expériences, je peux imaginer comment il peut t'être difficile de te rappeler quelque chose qui t'a procuré un sentiment de bien-être, quelque chose qui t'a fait du bien ". II répond : "Oui, et c'est pour ça que j'aime la drogue ».

Je savais que Mike consommait de la drogue à I'occasion. Je me suis tournée vers lui et j'ai dit : "Mike, qu'est-ce qui te procure un sentiment de bien-être ? "Mike répondit qu'il ne le savait pas mais qu'il avait souvent été battu lui aussi. "Je n'étais pas pour accepter ça... je me suis donc sauvé. Je ne retournerai jamais là. - J'aime la Floride ". Je dis : "Qu'est-ce qui te plaît en Floride? " II répond : "La plage ». Je dis : "Qu'est-ce qu'il y a d'agréable sur la plage ? " II se met à rire et répond : "Les filles, les corps, le... ". Je dis : "Est-ce tout ? " II répond que non, qu'il aime s'y sentir libre et que personne ne le bouscule. Je dis : "Pourquoi la plage te fait-elle te sentir libre? Je gage que tu y es bien parce que tu t'y sens libre ». Mike répond : "Je ne sais pas mais j'aime prendre du soleil. J'aime regarder l'eau ". Je dis : "Gary, Mike, la vie a dû être terrible pour vous deux à la maison, vous faire battre tout le temps. Je vous ai entendus raconter des problèmes qui se ressemblent et votre façon de vous en sortir se ressemble également, $c^{\prime}$ est-à-dire prendre de la drogue et aimer la plage. Les autres, qu'est-ce que vous en pensez? " J'ai, à ce moment, essayé d'obtenir des réponses du groupe. La plupart se sont ralliés à Gary et ont convenu que la drogue était le meilleur moyen de s'en sortir. Je dis : "Bien, puisque la plupart d'entre vous ont essayé le moyen de Gary pour résoudre le problème, pourquoi ne pas essayer celui de Mike? Que pensez-vous de demander la permission de tenir une rencontre sur la plage, le soir, alors que le lieu est désert et silencieux? Nous pourrions nous asseoir sur le sable, écouter l'océan, profiter du vent, du ciel et de l'air frais. Qu'en pensez-vous ? "Ils ont répondu que n'importe quoi était mieux que de s'asseoir ici mais "ils ne nous laisseront jamais aller ». Je dis : «Laissez-moi essayer d'obtenir la permission et peut-être verronsnous comment la plage pourrait nous aider à nous sentir bien ».

Cet exemple de pratique illustre une orientation à la fois humanitaire et de réadaptation présentée par la professionnelle qui mise sur la permission d'une randonnée sur la plage que l'agence accordera probablement. La professionnelle exprime, par son comportement, son appui au but de l'agence de provoquer le changement mais invite les membres à exercer leur propre pouvoir de décision afin d'arriver à une meilleure solution. Son attitude témoigne aussi d'une dose inhabituelle de patience, dont on a déjà noté la nécessité, pour lutter contre la contagion du groupe et contre sa résistance au 
changement; elle témoigne en outre du doute qui fait que l'on se demande si le jeu en vaut la chandelle (Bernstein, 1988).

L'orientation autoritaire et de contrôle, qui exige le changement de comportement, met l'accent sur la déviance des membres. Cette étiquette doit être contrebalancée par une position humaniste et de réadaptation, la stratégie étant d'aider les membres à découvrir qu'ils ont des besoins et qu'ils possèdent des caractéristiques communes aux autres humains (Bernstein, 1988). Le travailleur social de groupe essaie donc de normaliser la perception que la personne a d'ellemême; en prenant la responsabilité de son propre comportement, chacun pourra s'attaquer aux tâches développementales qui s'imposent pour recouvrer la santé à travers la vie du groupe. La stratégie de normalisation jette les bases nécessaires à la croissance de la confiance en soi et à une nouvelle sensibilité de chacun à l'effet qu'il produit sur les autres. La normalisation est la clé de voûte de l'aide mutuelle dans le groupe.

Pour le praticien, le dilemme consiste à n'être jamais libre de répondre ou non aux demandes autoritaires et de contrôle - peu importe le lieu où il rencontre un contexte d'autorité - même si, du point de vue professionnel, ce dernier est en conformité avec I'orientation humanitaire et de réadaptation. II doit appuyer les comportements exigés comme il le fait pour la présence obligatoire aux rencontres. Pour lui, la différence se situe dans l'aide qu'il apporte et qui permet aux membres d'assumer la responsabilité de leur propre changement et partant, de connaître une expérience de réalisation de soi et de confiance en soi.

La tâche du travailleur social à l'intérieur de l'organisme n'est pas de défier le pouvoir mais de découvrir des moyens d'atteindre les mêmes buts dans un milieu plus humanisé. Le professionnel peut et devrait communiquer, sans violer la confidentialité, la difficulté et la douleur que les membres connaissent souvent en essayant d'adopter le comportement exigé. Le discours de l'organisme sur la réadaptation doit être recherché et utilisé comme cadre qui permet de légitimer le plaidoyer en faveur des membres et le changement de climat de l'institution.

Dans tout contexte d'autorité, un certain degré de coexistence des orientations de réadaptation et de contrôle permet une souplesse potentielle des objectifs liés au changement de comportement et des moyens pour y arriver. L'attitude professionnelle du praticien devrait être d'évaluer continuellement la nature de ces orientations et leur effet sur l'organisation; il devrait établir les circonstances où elles sont le plus et le moins mises en pratique, déterminer les lieux où il peut appuyer la position humanitaire et de réadaptation et modifier la rigidité de l'orientation autoritaire et de contrôle. Reconnaître la 
coexistence $d^{\prime} u n$ certain équilibre entre les deux positions dans tout contexte d'autorité est essentiel à une pratique efficace lorsque la maîtrise ou le changement de comportement est obligatoire.

\section{Règles et règlements officiels}

On peut facilement reconnaître un contexte d'autorité par la prépondérance qu'il accorde aux directives, aux règles, aux règlements et aux rituels qui dictent ce qui est permis et ce qui est défendu aux membres comme au professionnel, et à l'obligation de s'y conformer. On peut s'attendre à ce que les milieux résidentiels qui possèdent une responsabilité de détention soient particulièrement soumis à des règlements rigides. Dans ces établissements, les règlements rigides et les règles imposés par les tribunaux, les services de protection de l'enfance et de probation, les commissions de libération conditionnelle, les bureaux des véhicules automobiles, etc., doivent être étroitement surveillés.

Le système officiel et bureaucratique de règlements et de règles est principalement conçu pour servir la fonction autoritaire et de contrôle. II est certain qu'il peut grandement entraver le développement du potentiel de groupe par des rites apparemment inutiles. Une simple demande de local et d'équipement peut nécessiter toute une paperasse avant que le professionnel et les membres puissent établir un plan des activités du groupe.

À cause de leur caractère obligatoire, les règles rigides augmentent la colère et la frustration des membres. Le professionnel acceptera cette colère tout comme il le fait pour les réactions au pouvoir d'imposer une assistance obligatoire et à la difficulté qu'éprouvent les membres par rapport au changement exigé. II les aidera à faire face encore une fois au pouvoir qui se cache derrière les règlements sans nier leurs propres responsabilités et les encouragera à revenir au travail de groupe pour découvrir ensemble ce qu'il peut leur offrir.

Le système puissant de règles n'est que rarement perçu de façon positive par le professionnel puisque, par sa nature même, le système diminue les occasions qu'il a de porter un jugement et d'exercer sa liberté professionnelle. II peut percevoir les règles liées au maintien de l'organisme comme étant assez peu crédibles. Le praticien est donc une proie facile pour les membres qui utilisent la manipulation pour les transgresser. Si le praticien succombe à la séduction de leurs arguments et se joint à leur action, ceux-ci auront réussi à le corrompre et à en faire la personnification d'une société injuste et malhonnête. De plus, I'influence du professionnel dans l'organisme sera grandement minée. 
L'intervention souhaitable du professionnel en réponse aux réactions des membres face à des règles défaillantes est, bien sûr, d'engager le groupe dans une sorte de processus légitime de changement social à l'intérieur de l'organisme. Le succès de tels efforts procure aux membres et au groupe un sentiment de satisfaction sur le plan de l'estime de soi et de l'affirmation personnelle. Une certaine innovation apportée à l'organisme peut de plus stimuler le désir de changement des membres (Garvin, 1988:260).

Des règles inflexibles peuvent devenir intolérables et être source de prolifération de contre-normes, incluant des codes propres aux détenus. Les contre-normes sont un phénomène de groupe qui demande, de la part du professionnel, la compréhension des processus négatifs de groupe et du pouvoir constructif de la vie de groupe. La connaissance technique de tels phénomènes, extérieurs à un groupe constitué et non volontaire, peut être la contribution du professionnel à l'effort d'un organisme disposé à éviter les injustices de la rigidité.

Les règlements et les règles, incluant les statuts légaux, peuvent servir à protéger le droit à la réadaptation des membres. Ils peuvent être invoqués par le travailleur social de groupe dans les négociations avec l'organisme pour assurer le renforcement de la position de réadaptation en contexte d'autorité. La connaissance des droits légaux peut aussi encourager les membres à s'engager avec le travailleur social dans des actions responsables pour appuyer la modification de règlements déraisonnables et inutiles, et à humaniser l'action de l'organisme sans affaiblir sa fonction légitime de contrôle. Alexander (1989 : 112) soulignant que la philosophie de réadaptation semble présentement avoir perdu des appuis dans les établissements correctionnels, note que la "11th Circuit Court of Appeals» a réaffirmé que : "le défaut des responsables de prisons d'assurer un niveau minimal de soins psychiatriques et de santé mentale aux prisonniers constitue une indifférence délibérée face aux problèmes médicaux urgents ». Alexander (1989: 111-112) recommande que les travailleurs sociaux en milieux correctionnels "rédigent un plan de traitement dans lequel les progrès et les changements seront documentés comme le demandent les stratégies de traitement". Cette pratique peut, au strict minimum, alimenter la plaidoirie faite au nom des membres en faveur de changements institutionnels mais elle peut aussi, le cas échéant, être le fondement d'une action légale.

\section{Personnel de surveillance et de contrôle}

Dans un contexte d'autorité, les rôles ont tendance à être hiérarchisés de façon bureaucratique et à être associés à l'une ou 
l'autre des polarités d'un continuum qui s'étend des fonctions humanitaires et de réadaptation à celles d'autorité et de contrôle. Cette situation prévaut tant dans les établissements correctionnels que dans un réseau d'agences communautaires interreliées. Les attitudes du personnel de surveillance et d'application des règles vont de l'allégeance rigide à l'institution, dictée par la préoccupation de maintenir la stabilité et l'autorité, jusqu'à la souplesse conditionnelle aux services humanitaires. Que l'on soit engagé de près ou de loin dans les évaluations critiques et les décisions relatives à la soumission et au changement obligatoire des membres, tout le personnel - incluant le travailleur social de groupe - éprouvera l'incertitude liée aux responsabilités de contrôle social en cause, comme dans le cas de la restauration du droit de visite d'un parent abuseur sexuel par exemple.

Le caractère officiel des relations avec le personnel diminue le degré de motivation et augmente celui d'incertitude, composantes qui favorisent les conflits au sein du personnel (Feldman, $1985: 303$ ). Une importante différenciation entre les membres $d^{\prime}$ un personnel dont la communication est limitée peut réduire les possibilités de surveillance et de mise en application des règles et permettre que des irrégularités passent inaperçues. Le professionnel et le groupe peuvent apprécier ces moments de plus grande autonomie, mais avec un certain risque, puisqu'à tout moment les règlements officiels sur lesquels le professionnel n'a aucun contrôle peuvent être invoqués par le personnel de surveillance. Un travailleur social de groupe avait, par exemple, offert des bonbons et des biscuits lors d'une réunion de prisonnières enceintes dans un centre de détention. Ayant appris que la règle qui l'interdisait n'était pas mise en application, il continua de le faire. Après plusieurs semaines, le règlement fut invoqué par un nouveau membre du personnel, et la nourriture, confisquée.

L'ensemble des rôles officiels du personnel exerce une puissante influence sur bon nombre d'aspects des processus de groupe, particulièrement sur les activités autres que la discussion. Garantir des ressources pour une activité éducationnelle qui demande un vidéo ou obtenir des laissez-passer pour une sortie peut exiger l'approbation de différents paliers administratifs rigides. La spontanéité et les activités non verbales seront bien sûr suspectes au personnel axé sur le contrôle, qui pourra les contrecarrer ou les étouffer. Quoique primordiales pour la vie du groupe, de telles activités présentent, aux yeux du personnel de surveillance, une menace à l'ordre établi et ajoutent peut-être un fardeau indésirable à la routine de contrôle.

Cependant, pour les membres dans un contexte d'autorité, les exercices non verbaux peuvent être un moyen judicieux de toucher 
leur méfiance profondément ancrée envers le personnel et le travailleur social.

\begin{abstract}
Après avoir procédé à un exercice de développement de la confiance avec un groupe de toxicomanes qui subissaient un traitement en résidence, le professionnel leur a demandé s'ils accordaient leur confiance aux autres par choix ou par nécessité. L'un des membres avoua qu'il ne faisait confiance à personne. Un autre répondit qu'il ne faisait pas confiance aux autres parce que ces derniers le laissaient tomber. Le travailleur social a alors demandé des exemples de telles situations et a voulu savoir si tout le monde était comme les gens de ces exemples. "Ouais, probablement, ... (pause) ...bien, peut-être pas tout le monde ». Le travailleur social leur a ensuite demandé s'il valait mieux ne pas faire confiance à tout le monde ou à la plupart des gens. La majorité des membres pensaient qu'on ne peut faire confiance à la plupart des gens. Leurs exemples portaient sur des détenteurs d'autorité. Le praticien a demandé aux membres s'ils croyaient que les autres leur faisaient confiance. La réponse a été unanime : tous croyaient qu'ils étaient dignes de confiance. La plupart ont ajouté que malgré cela, le personnel ne leur accordait pas particulièrement sa confiance.
\end{abstract}

Ce type d'activités de groupe permet au travailleur social d'amener les membres à transiger avec la méfiance qu'ils peuvent ressentir, et permet aux membres de développer différentes réponses à fournir aux autorités et de reconnaître leurs propres façons stéréotypées de percevoir les autres.

Comme il a déjà été mentionné, les clients à qui l'on impose une obligation en ressentent une intense colère et le personnel en contexte d'autorité alimente cet état. Les membres ressentent une profonde colère envers tout le personnel, professionnel ou autre, qui exerce une fonction de contrôle de façon brutale et capricieuse, ou qui semble trop faible ou inapte à les protéger.

L'expression de colère dans le groupe est une excellente occasion pour les membres de travailler à régler leur problème de perte de contrôle qui peut, d'une certaine façon, être lié à celui qui les a conduits à cet organisme. Le professionnel peut et devrait aider les membres et le groupe à explorer la situation : démontrer d'abord la justesse de leur colère; déterminer avec chacun les points qui peuvent déclencher la perte de contrôle; explorer les autres moyens d'exprimer leur colère; les préparer, par des mises en situation, à des rencontres comportant des aspects susceptibles de soulever leur colère. Le pouvoir dans un groupe réside dans l'aide mutuelle que les membres s'accordent et l'enrichissement personnel qui peut en découler. Cette expérience peut être nouvelle pour eux et unique dans un contexte d'autorité. 
La pratique liée aux réactions des membres face au personnel qui travaille dans un contexte d'autorité requiert une capacité d'écoute introspective (Goldstein, 1988) pour trouver, de l'intérieur vers l'extérieur, la signification que les membres accordent à l'expérience sans empêcher l'expression de sentiments négatifs. Le professionnel n'a pas à défendre les injustices causées par le personnel, mais doit plutôt aider les membres à examiner leurs perceptions de la réalité et leur accorder le bénéfice de celles qui sont exactes, même si la réparation de l'injustice est impossible. Dans ce cas, il démontre aux membres la portée des actions susceptibles d'être accomplies par luimême et par le groupe. En établissant des relations avec le personnel, le professionnel témoigne de l'habileté nécessaire à une perception différenciée des gens et à l'établissement de liens de travail réalistes.

La complexité de la structure de l'organisme fait resurgir le dossier de la confidentialité. Qu'est-ce que le travailleur social doit partager et avec qui ? Certaines questions difficiles sont posées par les membres à ce sujet pour vérifier les limites du pouvoir, consenti au professionnel, de dévoiler ou non l'information à l'organisme. Les membres s'attendent à ce qu'il clarifie ce qu'il entend partager avec le personnel officiel. Les renseignements relatifs à ce qui peut le placer ou placer les autres en danger seront fournis au personnel approprié. La confidentialité relative aux attitudes, aux expériences personnelles et aux sentiments passés - fâcheux ou négatifs - sera respectée; celle qui vise les compte rendus sur les changements de comportements liés aux buts et au maintien des règles et des règlements n'est pas aussi claire, ces compte rendus faisant partie d'une zone grise qui représente un dilemme pour le professionnel. Aucune règle de conduite ne peut être imposée si ce n'est le jugement du professionnel sur ce qui est bénéfique, du point de vue thérapeutique et éthique, et sur les risques que les décisions comportent.

La plupart des conflits que connaît le travailleur social de groupe naissent de ses rapports avec le personnel responsable de l'autorité et du contrôle; toutefois, ses relations avec le personnel dont la fonction est humanitaire et de réadaptation peuvent aussi poser des problèmes. Les membres qui se rendent compte du statut peu élevé du travailleur social de groupe dans la hiérarchie des fonctions de réadaptation peuvent essayer de le manipuler en se référant aux idées contraires de "leur psychiatre " ou de "leur répondant". Les membres ont une sensibilité étonnante à la compétition qui existe entre les divers personnels d'un organisme en autorité et l'utilisent pour augmenter leur pouvoir. Savoir manier l'humour, éviter le contre-transfert, reconnaître la manipulation du groupe comme autre tentative d'établir un meilleur équilibre du pouvoir entre les membres, l'organisme et le professionnel, voilà autant d'habiletés que le 
travailleur social doit posséder pour alimenter des relations efficaces avec le personnel et pour construire un groupe de travail social. L'habileté à reconnaître et à contrer les forces de compétition au sein du personnel est vitale pour améliorer et renforcer les éléments de réadaptation dans un contexte d'autorité.

Traditionnellement, dans la théorie du travail social de groupe, le rôle de médiation a été reconnu comme étant approprié et signifiant pour le praticien. Point $n^{\prime}$ est besoin de souligner qu'il existe des occasions d'actualiser ce rôle dans un contexte d'autorité où l'on trouve un personnel de surveillance et d'application des règles. On doit se rappeler que ce rôle requiert une acceptation officielle ou non de la part de toutes les parties en cause et la volonté de chacun de confier ce rôle au praticien. Une médiation réussie représente, pour les membres et pour l'organisme, un moyen de résoudre des problèmes autrement que par le seul exercice du pouvoir.

\section{Conclusion}

En terminant, nous désirons préciser que nous avons, dans le présent exposé, essayé de montrer qu'une position souple et relativiste est nécessaire au travailleur social de groupe qui œuvre en contexte d'autorité. Un praticien qui adopte une position absolue devient très vulnérable à une cooptation par le système d'autorité et de contrôle. Ce praticien, qui recherche la certitude, s'éloigne de la perspective humanitaire et de réadaptation du travail social de groupe et sacrifie les principes fondamentaux de la méthode.

Nous avons été séduites par les mots de Schlesinger (1989:41) : "L'absolutisme est abstrait, moniste, déductif, solennel et intimement lié à la déférence envers l'autorité. Le relativisme est concret, pluraliste, inductif, sceptique et intimement lié à la déférence envers l'expérience $[\ldots]$ ». Schlesinger poursuit : "L'absolutisme est finalement le grand ennemi de la lettre et de la vie de l'esprit».

Nous avons apporté une réponse affirmative à la question de savoir si le travail social de groupe peut fonctionner dans ce secteur si étranger à ses principes de base. Le travailleur social de groupe est le détenteur de l'orientation humaniste et de réadaptation dans son travail avec des groupes dont la participation des membres est obligatoire dans un contexte d'autorité. Non seulement le travail de groupe peut-il être pratiqué, mais les connaissances, les valeurs et les habiletés qu'il nécessite peuvent augmenter l'efficacité de la fonction de contrôle social par son défi envers l'absolutisme.

Même si notre réponse est affirmative, nous n'avons qu'effleuré cette question qui devra être maintes fois reprise dans les contextes particuliers de la pratique. Les habiletés liées aux relations interper- 
sonnelles qu'utilise le travailleur social de groupe en contexte d'autorité sont un cadeau offert à ceux et celles qui y sont engagés à titre de clients ou de membres non volontaires ou à titre de personnel de contrôle. Nous ne connaissons aucune autre discipline dans un milieu de service humanitaire pour qui l'interaction soit plus présente - cette interaction étant inhérente à la discipline - et plus profondément viable.

\section{Notes}

${ }^{1}$ Conférence prononcée lors du $11^{\mathrm{e}}$ symposium annuel de l'Association pour l'avancement du service social des groupes, tenu à Montréal en octobre 89. Charlotte Gagné en a fait la traduction.

${ }^{2}$ Les divers passages cités dans cet article ont été traduits d'ouvrages publiés en anglais.

\section{Références}

Alexander, Rudolph Jr. (1989). "The Right to Treatment in Mental and Correction Institutions ", Social Work, vol. 34, n 2 : 109-112.

Beninati, Joseph (1989). "Pilot Project for Male Batterers", Social Work With Groups, vol. 12, $\mathrm{n}^{\circ} 2:$ 63-74.

BERLINER, Arthur K. (1987). "Group Counseling With Alcohol Offenders : An Analysis and Typology of DWI Probationers", Social Work With Groups, vol. $10, \mathrm{n}^{\circ} 1: 17-32$.

BernstelN, Saul B. (1988). [Mimeo] "What Happened to Self-Determination ?" Baltimore. (À publier dans Proceedings of the Tenth Annual Symposium of the Association for the Advancement of Social Work With Groups).

BREKKE, John S. (1989). "The Use of Orientation Groups to Engage Hard-toReach Clients : Model, Method, and Evaluation", Social Work With Groups, vol. 12, n' 2 : 75-88.

BRENNEN, Nancy J. et Robert W. WeAKLAND (1983). "Assertiveness Training for Convicted Felons »: 342-360, dans N. Goroff (éd.), Reaping From The Field From Practice To Principle. Proceedings of Social Group Work Symposium Three - 1981. Hebron, Conn. : Practitioners Press.

BREton, Margot (1979). "Helping Abusive Families Through The Use of Small Groups ": 241-254, dans Sonia et Paul Abels (éd.), Social Work With Groups Proceedings 1979 Symposium. Louisville, Ky. : Committee for the Advancement of Social Work With Groups.

Citron, Peggy (1978). "Group Work With Alcoholic, Polydrug-involved Adolescents With Deviant Behavior Syndrome", Social Work With Groups, vol. 1, $\mathrm{n}^{\circ} 1: 39-52$.

EMERSON, Richard (1962). "Power-dependence relations", American Sociological Review, vol. 27, $\mathrm{n}^{\circ} 1$.

EpHross, Paul H. et Thomas V. VASSIL (1988). Groups That Work : Structure and Process. New York: Columbia University Press. 
Farley, Dennis et Judith MAGILl (1988). "An Evaluation of a Group Program For Men Who Batter ", Social Work With Groups, vol. 11, $\mathrm{n}^{\circ} 3$ : 53-65.

Feldman, Ronald A. (1985). "Group Work With Anti-social Youths » : 473-493, dans M. Sundel, P. Glasser, R. Sarri et R. Vinter (éd.), Individual Changes Through Small Groups. (2 $2^{\mathrm{e}}$ édition).

FinN, Jerry (1985). "Men's Domestic Violence Treatment Groups : A State-wide Study ", Social Work With Groups, vol. 8, n 3 : 81-94.

GARLAND, James, Hubert JONES et Ralph Kolodny (1970). "A Model for Stages of Development in Social Work Groups »: 17-71, dans S. Bernstein (éd.), Explorations in Group Work : Essays in Theory and Practice. Boston, Mass.

GarviN, Charles (1988). "Working With Groups in Social Control Situations " : 254-268, dans Englewood Cliffs (éd.), Contempory Group Work. ( $2^{\mathrm{e}}$ édition). New Jersey : Prentice Hall.

Geller, Janet A. (1978). "Reaching the Battering Husband", Social Work With Groups, vol. 1, $\mathrm{n}^{\circ} 1: 27-37$.

Glassman, Urania et Lenard Kates (1982). "Authority Themes and WorkerGroup Transactions : Additional Dimensions to the Stages of Development ": 947-967, dans Lang et Marshall (éd.), Patterns in the Mosaic. Proceedings 1982 Fourth Annual Symposium. Toronto, Canada : University of Toronto Press.

Goldstein, Howard (1988). "A Cognitive-Humanistic Social Learning Perspective on Social Group Work Practice », Social Work With Groups, vol. 11, $n^{\text {os }}$ 1-2: 9-32.

HASENFELD, Yeheskal (1985). "The Organizational Context of Group Work »: 294-309, dans M. Sundel, P. Glasser, R. Sarri et R. Vinter (éd.), Individual Change Through Small Groups. ( $2^{\mathrm{e}}$ édition). New York: The Free Press.

LANG, Norma (1972). "A Broad Range Model of Practice in Social Work Group ", Social Service Review, vol. 46, n 1 : 76-89.

LANG, Norma (1986). "Social Work Practice in Small Social Forms", Social Work With Groups, vol. 9, n" $4: 7-32$. Édition spéciale : Collectivity in Social Group Work : Concept and Practice.

Lance, Janice, Judy Barton et Susan Lanham (1981). "The Wiser Way: A Cognitive-Behavioral Model for Group Social Skills Training With Juvenile Delinquents ", Social Work With Groups, vol. 4, $n^{\text {os }}$ 3-4 : 37-48.

Levine, Baruch (1979). Group Psychotherapy: Practice and Development. Englewood Cliffs, New Jersey: Prentice Hall.

LeVINE, Baruch et Virginia Gallogly (1985). Group Therapy With Alcoholics. Beverly Hills : Sage Publications.

MILTON, Bob (1981). "Group Working at St. Charles", Social Work With Groups, vol. 4, $\mathrm{n}^{\text {os }} 1-2: 45-61$.

Nightencale, Jane (1989). "Discharge Planning : Promoting Patient Involvement Through Group Process", Social Work With Groups, vol. 13, n 2.

Nosko, Anne et B. Wallace (1988). "Group Work With Abusive Men : A Multidimensional Model ", Social Work With Groups, vol. 11, n $3: 33-52$.

Panepinto, William, James Garret, William Williford et John Priebe (1982). "A Short-Term Group Treatment Model for Problem-Drinker Drivers ", Social Work With Groups, vol. 5, n० $1: 33-40$.

PaPell, Catherine et Beulah Rothman (1980). "Relating the Mainstream Model of Social Work With Groups to Group Psychotherapy and the Structured Group ",Social Work With Groups, vol. 3, n 2 : 5-23. 
PRUDY, Frances et Norm NiCKLE (1981). "Practice Principles for Working With Groups of Men Who Batter ", Social Work With Groups.

San Francisco Chronicle, 9 novembre 1989 : 111-122.

SCHLESINGER, Arthur M. Jr. (1989). "The Perils of Absolutism »: 37-42, dans Brown Alumni Monthly (May), Brown University.

SCHWARTZ, William (1961). "The Social Worker in the Group " : 146-177, dans The Social Welfare Forum. New York : Columbia University Press.

SHIELDS, Sally A. (1986). "Busted and Branded : Group Work With Substance Abusing Adolescents in Schools ", Social Work With Groups, vol. 8, n 4 : 61-81.

SHULMAN, Lawrence (1988). "Groupwork Practice With Hard to Reach Clients : A Modality of Choice ", Groupwork, vol. 1, no $1: 5-16$. London : Whtiting Birch Ltd.

SMiTH, Thomas Edward (1985). "Groupwork with Adolescent Drug Abusers ", Social Work With Groups, vol. 8, $\mathrm{n}^{\circ} 1: 55-64$.

VINTER, Robert D. (1974). "Essential Components of Social Group Work Practice »: 9-34, dans P. Glasser, R. Sarri et R. Vinter (éd.), Individual Change Through Small Groups. New York : Free Press.

TuChman, Barbara W. (1982). Practicing History. New York : Ballantine Books. 